\title{
A SIMPLE EXAMPLE OF A UNIVERSAL SCHWARTZ SPACE
}

\author{
DANIEL J. RANDTKE
}

\begin{abstract}
A Schwartz space $E$ is universal if every Schwartz space is topologically isomorphic to a linear subspace of some power $E^{I}$ of $E$. In this paper concrete examples of universal Schwartz spaces are exhibited.
\end{abstract}

In [2, Theorem 8, p. 800] J. Diestel, S. A. Morris and S. A. Saxon announced that there exist universal Schwartz spaces and indicated that a concrete example of such a space had not as yet been exhibited. In this paper we exhibit three such spaces the simplest of which is the following: Take the Banach space $c_{0}$ of zero-convergent scalar valued sequences and equip $c_{0}$ with the topology $\mathscr{S}$ defined by seminorms of the form

$$
q_{\lambda}(\mu)=\sup \left|\lambda_{n}\right|\left|\mu_{n}\right|
$$

where $\lambda$ ranges over all of the elements of $c_{0}$. The space $c_{0}$ equipped with the topology $\mathscr{S}$ is then a universal Schwartz space. (For the definition and basic properties of Schwartz spaces we refer the reader to [3, Chapter 3 , §15, pp. 271-288].)

If $E$ is a locally convex space (all of our spaces are assumed to be Hausdorff), then $\tau\left(E, E^{\prime}\right)$ denotes the Mackey topology on $E$ with respect to the dual system $\left\langle E, E^{\prime}\right\rangle$.

A seminorm $q$ on a locally convex space $E$ is precompact if there is a sequence $\lambda$ in $c_{0}$ and an equicontinuous sequence $\left\{a_{n}\right\}$ in the topological dual $E^{\prime}$ of $E$ such that, for each $x$ in $E$,

$$
q(x) \leqq \sup \left|\lambda_{n}\right|\left|\left\langle x, a_{n}\right\rangle\right| \text {. }
$$

(For the basic properties of precompact seminorms we refer the reader to [6].) A linear operator $T: E \rightarrow F$ from one locally convex space into another is precompact [respectively, compact] if $T$ transforms a neighborhood of 0 in $E$ into a precompact [respectively, relatively compact] subset of $F$. In [6, Corollary 2.10] we showed that a linear operator

Received by the editors March 13, 1972 and, in revised form, June 16, 1972.

AMS (MOS) subject classifications (1970). Primary 46A05.

Key words and phrases. Schwartz space, universal Schwartz space, precompact seminorm, precompact operator, compact operator.

(c) American Mathematical Society 1973 
$T: E \rightarrow F$ from one normed space into another is precompact if and only if there is a precompact seminorm $q$ on $E$ such that, for each $x$ in $E$, $\|T x\| \leqq q(x)$. Terzioglu [8, (4), p. 237] (and, independently, the author $[6,3.1$, p. 95]) has shown that: A locally convex space $E$ is a Schwartz space if and only if each continuous seminorm on $E$ is precompact.

Let $l_{\infty}$ denote the Banach space (sup norm $\|\cdot\|_{\infty}$ ) of bounded scalar valued sequences and let $C[0,1]$ denote the Banach space (sup norm) of continuous scalar valued functions on the unit interval $[0,1]$.

A projective limit proj $\lim f_{\mu \nu}\left(E_{v}\right)$ of Banach spaces $E_{v}$ (for the definition of projective limit see [3, p. 155]) is compact if for each $\mu$ there is a $\nu>\mu$ such that the linear operator $f_{\mu v}: E_{v} \rightarrow E_{\mu}$ is compact. If each $E_{v}=E$, then we say that proj $\lim f_{\mu \nu}\left(E_{v}\right)$ is a projective limit of $E$-spaces and write proj $\lim f_{\mu \nu}(E)$. In [7, Theorem 2.4] we showed that: If $E$ denotes any one of the spaces $c_{0}, l_{\infty}$ or $C[0,1]$ then a locally convex space $F$ is a Schwartz space if and only if $F$ is topologically isomorphic to a linear subspace of a compact projective limit of $E$-spaces.

1. Proposition. Let $E$ be a locally convex space.

If $\mathscr{S}$ denotes the topology on $E$ defined by the family of $\tau\left(E, E^{\prime}\right)$-precompact seminorms on $E$, then $\mathscr{S}$ is the strongest Schwartz topology on $E$ compatible with the dual system $\left\langle E, E^{\prime}\right\rangle$.

Proof. Clearly $\mathscr{S}$ is compatible with the dual system $\left\langle E, E^{\prime}\right\rangle$.

To show that $E[\mathscr{S}]$ is a Schwartz space, it suffices (see $[8,(4), \mathrm{p} .237]$ or $\left[6,3.1\right.$, p. 95]) to show that if $\lambda$ is a sequence in $c_{0}$ and $\left\{a_{n}\right\}$ is a $\tau\left(E, E^{\prime}\right)$ equicontinuous sequence in $E^{\prime}$, then there is a sequence $\mu$ in $c_{0}$ and an $\mathscr{S}$-equicontinuous sequence $\left\{b_{n}\right\}$ in $E^{\prime}$ such that, for each $x$ in $E$,

$$
\sup \left|\lambda_{n}\right|\left|\left\langle x, a_{n}\right\rangle\right| \leqq \sup \left|\mu_{n}\right|\left|\left\langle x, b_{n}\right\rangle\right| \text {. }
$$

If $\lambda$ is a sequence in $c_{0}$ and $\left\{a_{n}\right\}$ is a $\tau\left(E, E^{\prime}\right)$-equicontinuous sequence in $E^{\prime}$, then choose sequences $\mu$ and $\tau$ in $c_{0}$ so that $\lambda_{n}=\mu_{n} \tau_{n}$ for all $n$. Let $b_{n}=\tau_{n} a_{n}$. Since

$$
\left\langle x, b_{n}\right\rangle \leqq \sup \left|\tau_{n}\right|\left|\left\langle x, a_{n}\right\rangle\right|
$$

it follows that $\left\{b_{n}\right\}$ is an $\mathscr{S}$-equicontinuous sequence in $E^{\prime}$ that satisfies (*). Therefore, $E[\mathscr{S}]$ is a Schwartz space and it is easy to see that $\mathscr{S}$ is the strongest Schwartz topology on $E$ compatible with the dual system $\left\langle E, E^{\prime}\right\rangle$.

2. THEOREM. Let $E$ denote any one of the spaces $c_{0}, l_{\infty}$ or $C[0,1]$.

If $\mathscr{S}$ denotes the topology on $E$ defined by the family of precompact seminorms on $E$, then $E[\mathscr{S}]$ is a universal Schwartz space.

Proof. Since the norm topology on $E$ coincides with the $\tau\left(E, E^{\prime}\right)$ topology on $E$, Proposition 1 implies that $E[\mathscr{S}]$ is a Schwartz space. 
Let $F$ be a Schwartz space. By [7, Theorem 2.4], $F$ is topologically isomorphic to a linear subspace of a compact projective $\operatorname{limit}$ proj $\lim f_{\mu \nu}(E)$ of $E$-spaces (where $\mu, \nu$ belong to a given directed set $I$ ). Let $T$ denote the natural embedding of $P=\operatorname{proj} \lim f_{\mu \nu}(E)$ into $E^{I}$ and let $S$ denote the identity operator from $E^{I}$ into $E[\mathscr{S}]^{I}$. Since $\mathscr{S}$ is weaker than the norm topology on $E, S$ is continuous. To complete the proof we show that $S T$ is open.

Let $\mu$ be an element of $I$ and let $U$ denote the set of all points $x$ in $P$ with $\left\|x_{\mu}\right\| \leqq 1$. Choose $\nu>\mu$ in $I$ so that $f_{\mu \nu}: E \rightarrow E$ is compact. Let $q$ be a precompact seminorm on $E$ such that $\left\|f_{\mu \nu}(x)\right\| \leqq q(x)$ for all $x$ in $E$. Let $V$ denote the set of all $x$ in $E[\mathscr{S}]^{I}$ such that $q\left(x_{\mu}\right) \leqq 1$, then it is easy to see that

$$
V \cap S T(P) \subset S T(U)
$$

Therefore, $S T$ is open and $F$ is topologically isomorphic to a linear subspace of $E[\mathscr{S}]^{I}$.

3. CoRollary. If $c_{0}$ is equipped with the topology $\mathscr{S}$ defined by seminorms of the form

$$
q_{\lambda}(\mu) \leqq \sup \left|\lambda_{n}\right|\left|\mu_{n}\right|
$$

where $\lambda$ ranges over all of the elements of $c_{0}$, then $c_{0}[\mathscr{S}]$ is a universal Schwartz space.

Proof. This follows from Theorem 2 above and the following consequence of [1, Theorem 2.4 , p. 477]:

If $q$ is a precompact seminorm on $c_{0}$, then

$(*)(*)$ there is a sequence $\lambda$ in $c_{0}$ such that, for each $\mu$ in $c_{0}$,

$$
q(\mu) \leqq \sup \left|\lambda_{n}\right|\left|\mu_{n}\right|
$$

4. REMARK. By using $(*)(*)$ above we have been able to show that every precompact linear operator $T: c_{0} \rightarrow E$ from $c_{0}$ into a normed space $E$ has a representation of the form $T(\mu)=\sum \lambda_{n} \mu_{n} y_{n}$ where $\lambda$ is a sequence in $c_{0}$ and $\left\{y_{n}\right\}$ is a (weakly) unconditionally summable sequence in $F$. Moreover, $c_{0}$ is the only Banach space with basis that has this property. The details will appear elsewhere.

In view of the main result of [6] we pose the following question: If an $\mathscr{L}_{\infty}$-space $E$ (see [4] and [5] for the definition and basic properties of $\mathscr{L}_{\infty}$-spaces) is equipped with the topology $\mathscr{S}$ defined by the family of precompact seminorms on $E$, is $E[\mathscr{S}]$ a universal Schwartz space? It has been conjectured (see [5, Problem 3d, p. 346]) that every infinite dimensional $\mathscr{L}_{\infty}$-space contains a subspace isomorphic to $c_{0}$. A positive answer to this conjecture would imply a positive answer to our question. 
The author would like to thank the referee for his comments and references.

\section{REFERENCES}

1. J. B. Conway, The strict topology and compactness in the space of measures. II, Trans. Amer. Math. Soc. 126 (1967), 474-486. MR 34 \#6503.

2. J. Diestel, S. A. Morris and S. A. Saxon, Varieties of locally convex topological vector spaces, Bull. Amer. Math. Soc. 77 (1971), 799-803.

3. J. Horváth, Topological vector spaces and distributions. Vol. I, Addison-Wesley, Reading, Mass., 1966. MR 34 \#4863.

4. J. Lindenstrauss and A. Pelczyński, Absolutely summing operators in $\mathscr{L}_{p}$-spaces and their applications, Studia Math. 29 (1968), 275-326. MR 37 \#6743.

5. J. Lindenstrauss and H. P. Rosenthal, The $\mathscr{L}_{p}$-spaces, Israel J. Math. 7 (1969), 325-349. MR 42 \#5012.

6. D. J. Randtke, Characterizations of precompact maps, Schwartz spaces and nuclear spaces, Trans. Amer. Math. Soc. 165 (1972), 87-101.

7. - A structure theorem for Schwartz spaces, Math. Ann. (to appear).

8. T. Terzioglu, On Schwartz spaces, Math. Ann. 182 (1969), 236-242. MR 40 \#683.

Department of Mathematics, University of Georgia, Athens, Georgia 30601 